\title{
Unconventional magnetic behavior of iron-oxide nanoparticles in polymeric matrices
}

\author{
A. M. Testa, ${ }^{\text {a) }}$ S. Foglia, L. Suber, and D. Fiorani \\ ICMAT-CNR, Area della Ricerca di Roma, Monterotondo Stazione, Rome, Italy
}

LI. Casas, A. Roig, and E. Molins

Institut de Ciència de Materials de Barcelona (ICMAB-CSIC), Campus UAB, 08193 Bellaterra, Catalunya, Spain

J. M. Grenèche

Laboratoire de Physique de l'État Condensé, UMR CNRS 6087, Universite du Maine, Faculté des Sciences, 72085 Le Mans Cedex 9, France

\section{J. Tejada}

Universitat de Barcelona, Xerox Laboratory for Magnetics Research, Avenue Diagonal 647, 08028 Barcelona, Spain

(Received 27 November 2000; accepted for publication 3 May 2001)

We report the results of magnetization and ${ }^{57} \mathrm{Fe}$ Mössbauer spectroscopy measurements performed in the temperature range $5-300 \mathrm{~K}$ on composites containing iron-oxide nanoparticles encased in polystyrene type resins. After carrying out a suitable field treatment in order to decouple the particles from the matrix, a fraction of the particles freely rotate in response to an applied magnetic field. (C) 2001 American Institute of Physics. [DOI: 10.1063/1.1383019]

\section{INTRODUCTION}

Significant progress has recently been made in producing nanocomposite magnetic materials with superior properties and potential important application. The common feature of such materials is an interface across which the magnetic interactions occur. The detailed nature of such interactions is attracting increased experimental and theoretical attention. Possible magnetic mechanisms operating at the interfaces include dipole-dipole, exchange (isotropic and anisotropic), superexchange, and magnetoelastic interactions. At least one of these mechanisms, and often more than one, are contemporarily operating and the type of such interactions depend not only on the microstructure, but also on whether itinerant or localized electrons are involved. As a result, a wide range of magnetization processes and properties can be observed depending on the specific composite system. Examples of current interest include magnetically hard and soft metallic alloys, ${ }^{1}$ core-surface nanoparticles, ${ }^{2}$ and nanocomposite films. ${ }^{3,4}$ Recently, a new magnetic behavior has been observed at low temperature $(5 \mathrm{~K})$ in insulating composites based on crystalline $\gamma-\mathrm{Fe}_{2} \mathrm{O}_{3}$ (maghemite) nanoparticles dispersed in a solid polymer matrix, ${ }^{5}$ prepared by freezing a methanol based ferrofluid of the composite: magnetic particles have been observed rotating freely in response to an applied static magnetic field (i.e., "free rotor behavior") after conditioning the material in a cycling magnetic field.

To check the existence of such behavior in other types of iron-oxide based nanocomposites, we investigated, in the temperature range 5-300 K, the magnetic properties of composite iron-oxide nanoparticles encased in matrices based on

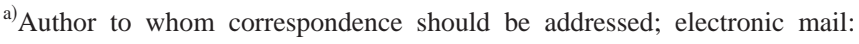
magnet@mlib.cnr.it commercial ion-exchange type polystyrene. Global magnetization measurements and Mössbauer spectrometry show that for the nanocomposites based on weak cationic exchangers (i.e., phosphonic resin), a free rotor-like behavior exists even at room temperature for at least a fraction of the particles.

\section{EXPERIMENT}

The composites containing iron-oxide nanoparticles were synthesized using commercial cation exchanger resins which were used as networks. For all the resins the matrix was polystyrene crosslinked with divinylbenzene. The resins labeled "BR-P" have a crosslinkage degree of $1 \%-2 \%$ and contain phosphonic functional groups; those labeled "RD-S" show a crosslinkage of $2 \%$ and contain sulphonic groups. The resins are formed by beads of $\sim 100-300 \mu \mathrm{m}$ in diameter, a single bead being made up of microglobules. Interstitial spaces between these microglobules form the macropores ${ }^{6}$ in which the iron-oxide nanoparticles can be fitted. The iron-oxide nanoparticles (magnetite, $\mathrm{Fe}_{3} \mathrm{O}_{4}$ ) synthesis was performed under $\mathrm{N}_{2}$ atmosphere, by dispersing the resin in a $\mathrm{Fe}^{3+} / \mathrm{Fe}^{2+}$ (2:1 ratio) chloride solution.

Matrix cations $\left(\mathrm{Na}^{+}\right.$or $\left.\mathrm{H}^{+}\right)$were exchanged with $\mathrm{Fe}^{3+}$ and $\mathrm{Fe}^{2+}$ ions. The exchange was followed by hydrolysis and polymerization in an alkaline medium at $65^{\circ} \mathrm{C}$ with formation of $\mathrm{Fe}_{3} \mathrm{O}_{4}$ nanoparticles in the resin macropores. To increase the iron-oxide nanocomposite loading, the process was repeated a number of times up to the desired loading value. After the first loading cycle the iron percentage, determined by atomic absorption spectrophotometry, was $\approx 3 \%$ and $11 \%$ for the BR-P and RD-S based samples, respectively.

Scanning electron microscopy as well as energy dispersive spectra (EDS) measurements were carried out with a Cambridge 360; Transmission electron microscopy (TEM) 


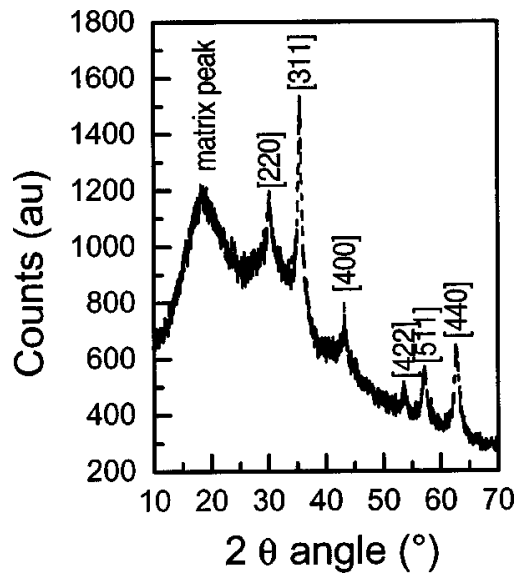

FIG. 1. XRD powders diffraction pattern of samples BR-P; magnetite peaks are indexed.

measurements were performed with a Philips CM 20 operating at $200 \mathrm{kV}$. Magnetization versus temperature $\left(T_{\max }\right.$ $=300 \mathrm{~K})$ and applied field $\left(H_{\max }=55 \mathrm{kOe}\right)$ measurements were performed with a commercial semiconducting quantum interference device magnetometer. Mössbauer spectra, without field and in a weak magnetic field $(0.4 \mathrm{kOe})$ applied perpendicular to the $\gamma$ beam were recorded with a conventional transmission spectrometer with a ${ }^{57} \mathrm{Co}$ source in a Rh matrix; in field $(60 \mathrm{kOe})$ spectra were recorded using a cryomagnetic device in which the external field was applied parallel to the $\gamma$ beam.

\section{RESULTS AND DISCUSSION}

\section{A. Structural and morphological properties}

$\mathrm{X}$-ray powder diffraction patterns were recorded between $4^{\circ}$ and $70^{\circ}$ of $2 \theta$ using a Siemens D-5000 diffractometer with $\mathrm{Cu} K_{\alpha}$ radiation. The diffraction pattern of the BR-P nanocomposite shows a very broad peak at $\sim 19^{\circ}$, due to the amorphous nature of the matrix and several broad peaks (Fig. 1) between $25^{\circ}$ and $45^{\circ}$. As $\gamma-\mathrm{Fe}_{2} \mathrm{O}_{3}$ and $\mathrm{Fe}_{3} \mathrm{O}_{4}$ reflections are very close to each other, $\mathrm{x}$-ray diffraction does not allow for unambiguous distinction between the two iron oxides.

TEM measurements show that in the BR-P nanocomposites [Fig. 2(a)] the iron-oxide nanoparticles are almost regularly spherical shaped, with a size distribution varying from 3 to $4 \mathrm{~nm}$ up to $15 \mathrm{~nm}$ in diameter. As the macropores have diameters of $\sim 0.5 \mu \mathrm{m}$, it is expected that particles and/or aggregates of particles of smaller size will fill the pores. The presence of aggregates with a diameter larger than $500 \mathrm{~nm}$ on the surface of the beads could not be excluded.

EDS elemental analyses on these aggregates show that the value of the $\mathrm{Fe} / \mathrm{O}$ atomic ratio is $\approx 0.77$, very close to the 0.75 expected value for $\mathrm{Fe}_{3} \mathrm{O}_{4}$. The BR-P based materials had a black color which changed to red-brown over time, revealing a progressive oxidation process towards maghemite. This was also confirmed by Mössbauer spectrometry (see Sec. III).

In the case of the RD-S based composite, TEM measurements evidenced spherical iron-oxide particles with an av-
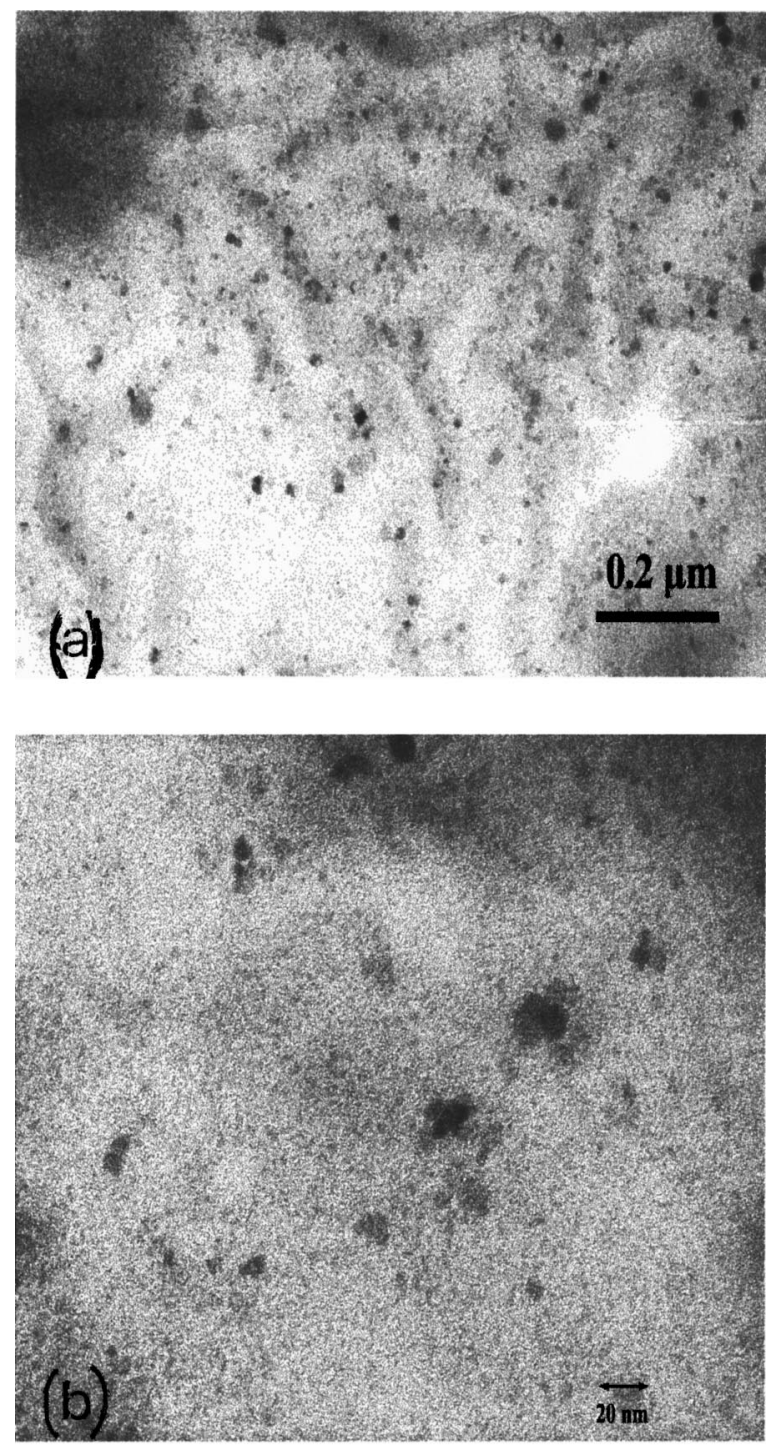

FIG. 2. TEM micrographs of samples BR-P (a) and RD-S (b).

erage diameter of $5 \mathrm{~nm}$ [Fig. 2(b)] while the XRD and EDS results ${ }^{7}$ indicated the formation of the stable $\gamma-\mathrm{Fe}_{2} \mathrm{O}_{3}$ as the major phase. In contrast with the BR-P based materials, the material just prepared has a red-brown color. For the RD-S based composite the smaller average size of the particles, and hence the increased surface to volume ratio, is likely to promote the oxidation process responsible for the maghemite formation, as previously observed with other preparation techniques. ${ }^{8}$

\section{B. Magnetic properties}

Magnetization versus temperature measurements performed after the zero field cooling (ZFC) and field cooling (FC) $\left(H_{a}=100 \mathrm{Oe}\right)$ processes, are reported in Fig. 3. The curves exhibit the typical features of an assembly of magnetic nanoparticles presenting a volume $(V)$ distribution, implying a distribution of anisotropy energy barriers $E_{B}\left(E_{B}\right.$ $=K_{a} V$ for uniaxial anisotropy) and, consequently, of relaxation times $\tau^{9}{ }^{9}$ For an assembly of noninteracting particles with a certain size distribution, the temperature of the ZFC susceptibility maximum $T_{m}$ is found to increase with the 


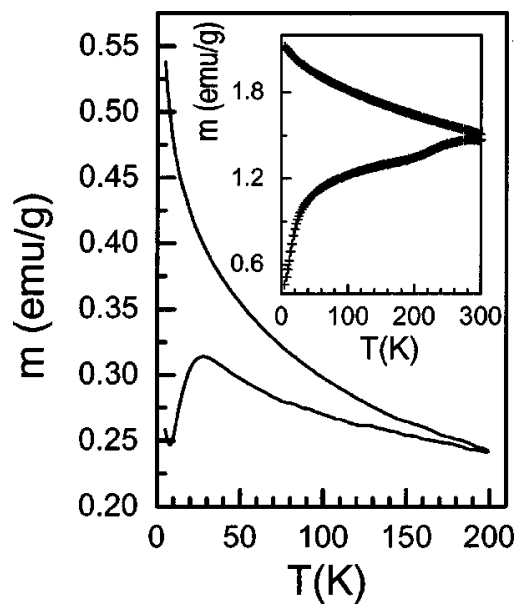

FIG. 3. Magnetization as a function of temperature for samples RD-S and BR-P (inset).

particle size and, in general, it is related to the average blocking temperature $\left\langle T_{B}\right\rangle$, according to relationships that depend on the volume distribution function. ${ }^{9}$ For the RD-S sample, the ZFC curve shows a maximum at $T_{m} \approx 30 \mathrm{~K}$. The FC curve splits from the ZFC one at $T \gg T_{m}$, revealing the existence of a broad distribution of particle size; moreover the FC curve follows a Curie-Weiss law, indicating that the sample consists of noninteracting or very weakly interacting particles. In contrast, the ZFC magnetization of the BR-P sample increases with temperature, with a change of slope at $\approx 200 \mathrm{~K}$; then it tends to flatten off around $\approx 300 \mathrm{~K}$, suggesting the presence of a broad maximum which develops beyond the highest temperature investigated. This indicates that the size distribution is shifted to larger sizes with respect to the RD-S sample, in agreement with TEM results. Moreover, the FC curve does not follow the Curie-Weiss law, suggesting the presence of much stronger interparticle interactions.

For both samples, magnetization versus field curves at low temperature shows an ordinary hysteretic behavior, with a time-dependent magnetization. This confirms the existence in the blocked state of metastable states, separated by energy barriers, which prevent a free magnetization reversal. Unconventional features in the magnetic properties of sample BR-P appear when the magnetic field is rapidly cycled from plus to minus $5 \mathrm{kOe}$ (magnetic training). Figure 4 shows the ZFC magnetization versus data measured at $5 \mathrm{~K}$ for the RD-S and BR-P samples after the magnetic training. Actually, the measurement consists of a sequence of minor loops performed as follows: in the first step, the field is increased up to a positive value $\left(H_{1}=100 \mathrm{Oe}\right)$, then the field is decreased down to a negative value $\left(H_{2}=-400 \mathrm{Oe}\right)$, larger than the positive previous one. Such a procedure is repeated for increasing amplitudes of the maximum field of the minor loop, $2 \mathrm{kOe}$ being the largest value of the applied field. For the RD-S sample the coercive field was found to increase with the strength of the applied field, as for ordinary hard magnetic materials, reaching a value of $\approx 130$ Oe at $H_{a}=2 \mathrm{kOe}$. Such a value has been taken as the average between the demagnetizing and remagnetizing branches of the hysteresis loop because of its asymmetry. In addition to different possible

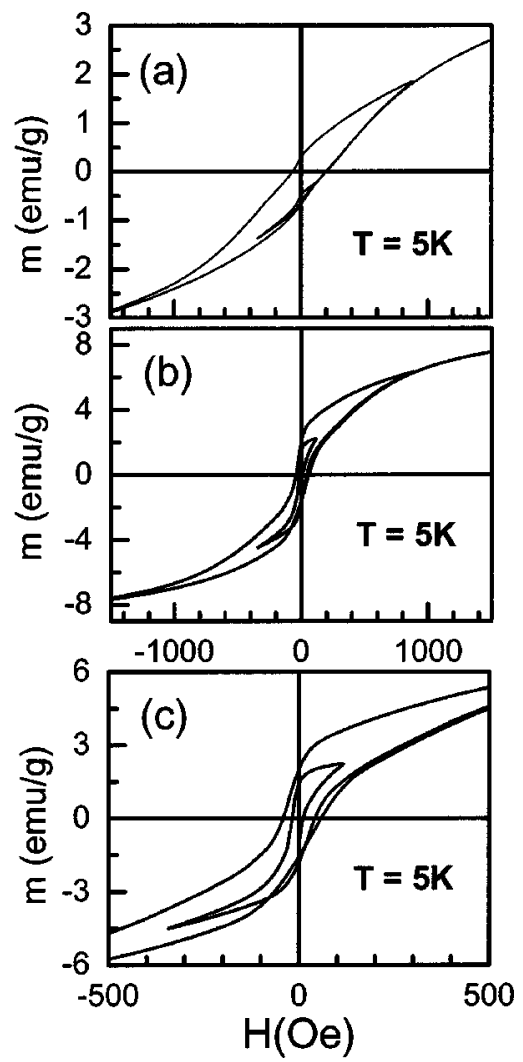

FIG. 4. Magnetization as a function of the applied magnetic field measured at $5 \mathrm{~K}$ after training in a magnetic field of $\pm 5 \mathrm{kOe}$ : (a) sample RD-S; (b) sample BR-P; (c) detail of $M$ vs $H$ in the range \pm 500 Oe for the sample BR-P.

physical reasons, the asymmetry should be primarily due to the finite remnant magnetization after the magnetic training. In contrast, for the BR-P sample, only a small coercive field $\left(H_{c} \approx 50 \mathrm{Oe}\right)$ was measured and a sharp drop in the magnetization was observed in the proximity of the zero applied field, followed by a continuous change in the magnetization. This suggests that a fraction of particle moments can freely rotate in response to magnetic fields of low amplitude. It is also observed that the hysteresis loop becomes narrower and narrower when the temperature increases. However, this cannot be directly related to the free rotor effect, due to the increasing contribution of unblocked particle moments and the expected decrease in the coercive field of blocked particles.

In order to have a microscopic insight into such unconventional behavior, we have finally undertaken Mössbauer spectrometry on the BR-P sample. In particular, measurements were carried out at 300,77 , and $4.2 \mathrm{~K}$ and with an applied field of $60 \mathrm{kOe}$ parallel to the $\gamma$-ray direction at 10 K. At $4.2 \mathrm{~K}$ [Fig. 5(a)] the Mössbauer spectrum is totally split into an asymmetrical sextet with broad lines. The fitting model, which requires at least two magnetic components, cannot give a single solution with accurate values of hyperfine parameters, making the phase identification ${ }^{10}$ difficult. Nevertheless, since there is no clear evidence for the divalent iron component, the data analysis suggests that $\mathrm{Fe}_{3} \mathrm{O}_{4}$ oxidized to $\gamma-\mathrm{Fe}_{2} \mathrm{O}_{3}$. At $T=10 \mathrm{~K}$ [Fig. 5(b)], the application of a $60 \mathrm{kOe}$ external field parallel to the $\gamma$-ray direction leads to 


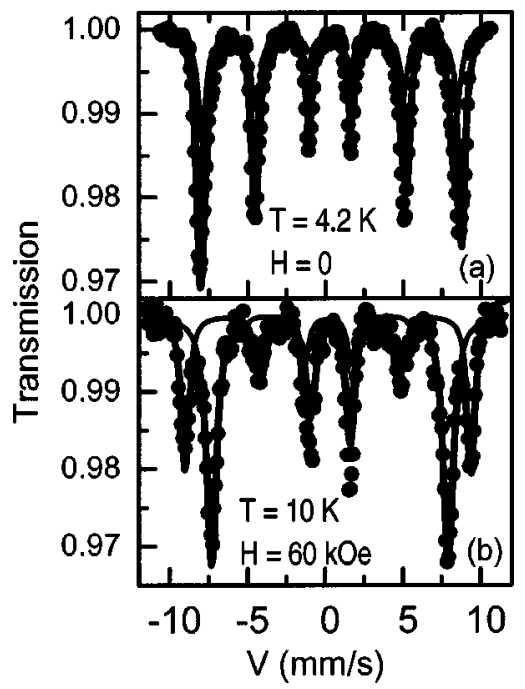

FIG. 5. Mössbauer spectra of sample BR-P: (a) $4.2 \mathrm{~K}, H_{a}=0$; (b) $10 \mathrm{~K}$ with $H_{a}=60 \mathrm{kOe}$.

a split of the magnetic spectrum into two resolved magnetic sextets, with rather low intensity intermediate lines. The refined values listed in Table I suggest a ferrimagnetic behavior of the compound. Indeed, one observes the significant increase (decrease) of the effective field $B_{\text {eff }}$ for the outer (inner) component compared to the hyperfine field $B_{\text {hyp }}$. Because their magnetic moment is opposite the hyperfine field, the values of effective field $B_{\text {eff }}$ and of $\beta$ which is defined as the angle comprised between $B_{\text {eff }}$ and $\gamma$-ray directions, give rise to the hyperfine field values of both tetrahedral and octahedral iron sites using the following relationship: $B_{\text {hyp }}^{2}$ $=B_{\text {eff }}^{2}+B_{\text {ext }}^{2}-2 B_{\text {ext }} B_{\text {eff }} \cos \beta$. The refined values of hyperfine parameters (Table I) are close to those previously reported for maghemite, ${ }^{11}$ confirming the occurrence of the oxidation process in the material. Spectra at $77 \mathrm{~K}$ [Fig. 6(a)] and at room temperature [Fig. 7(a)] consist of a superposition of a six-line Zeeman spectrum and a central doublet. The two components correspond to the magnetically blocked and superparamagnetic particles, respectively. At $77 \mathrm{~K}, 33 \%$ of the total resonant area corresponds to the unblocked particles. To find evidence of chemical decoupling of the particles from the matrix, a series of in-field spectra have also been performed without removing the sample from the spectrometer. Several spectra were recorded at $77 \mathrm{~K}$ according to the following sequence, which should mimic the effect of the "magnetic training" carried out before the magnetization measurements:

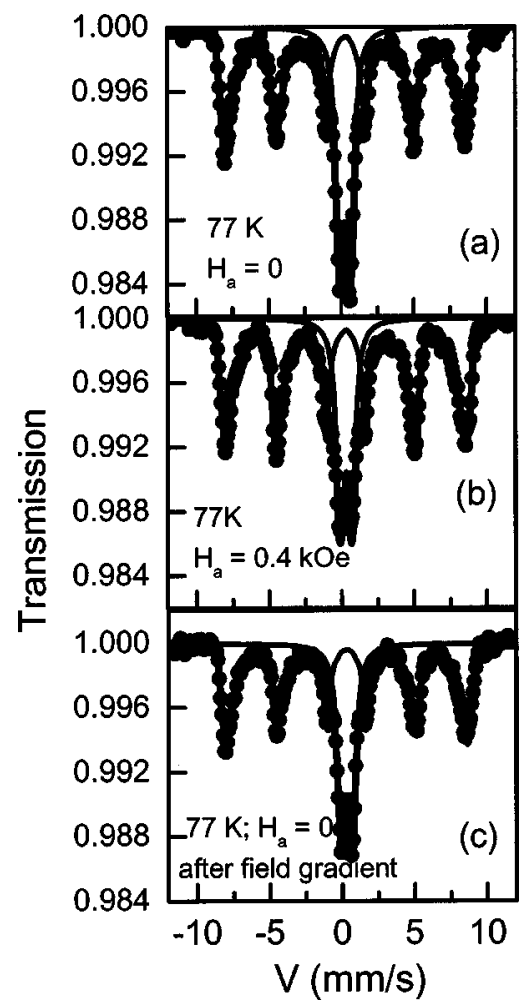

FIG. 6. Mössbauer spectra of sample BR-P at $77 \mathrm{~K}$ : (a) $H_{a}=0$; (b) $H_{a}$ $=0.4 \mathrm{kOe}$; (c) $H_{a}=0$ after the magnetic field gradient exposure.

(i) spectrum recorded in the absence of an applied magnetic field [Fig. 6(a)];

(ii) the sample was exposed to a field gradient of low intensity $(0.4 \mathrm{kOe})$, where the angle between the magnetic field and the sample changed from $45^{\circ}$ to $135^{\circ}$;

(iii) spectrum recorded on sample exposed to a field of 0.4 kOe applied perpendicular to the $\gamma$ beam [Fig. 6(b)]; and

(iv) zero field spectrum recorded after removing the field [Fig. 6(c)].

The geometrical conditions (source-sample and sampledetector distances) were kept constant to avoid solid-angle effects.

It is observed that the application of a small magnetic field increases the magnetic six-line subspectra at the expense of the superparamagnetic doublet as well as the ordering of particle moments along the magnetic field. This implies a large low field susceptibility, which is consistent with

TABLE I. Mössbauer hyperfine parameters of sample BR-P.

\begin{tabular}{lccccccc}
\hline \hline & Site & $\delta_{\mathrm{Fe}}(\mathrm{mm} / \mathrm{s})$ & $\begin{array}{c}2 \epsilon \\
(\mathrm{mm} / \mathrm{s})\end{array}$ & $B_{\text {eff }}(\mathrm{T})$ & $B_{\text {hyp }}(\mathrm{T})$ & $\beta\left(^{\circ}\right)$ & Rel. area \\
\hline $\begin{array}{l}T=4.2 \mathrm{~K} \\
H=0\end{array}$ & tetr. Fe & 0.37 & 0.00 & - & $49.7(4)$ & - & $38(5) \%$ \\
& oct. Fe & 0.51 & 0.00 & - & $52.0(4)$ & - & $62(5) \%$ \\
$T=10 \mathrm{~K}$ & tetr. Fe & $0.37(2)$ & $0.00(1)$ & $56.9(4)$ & $51.2(4)$ & $18(5)$ & $29(5) \%$ \\
$H=60 \mathrm{kOe}$ & oct. Fe & $0.51(2)$ & $0.02(1)$ & $47.0(4)$ & $52.1(4)$ & $43(5)$ & $71(5) \%$ \\
\hline \hline
\end{tabular}




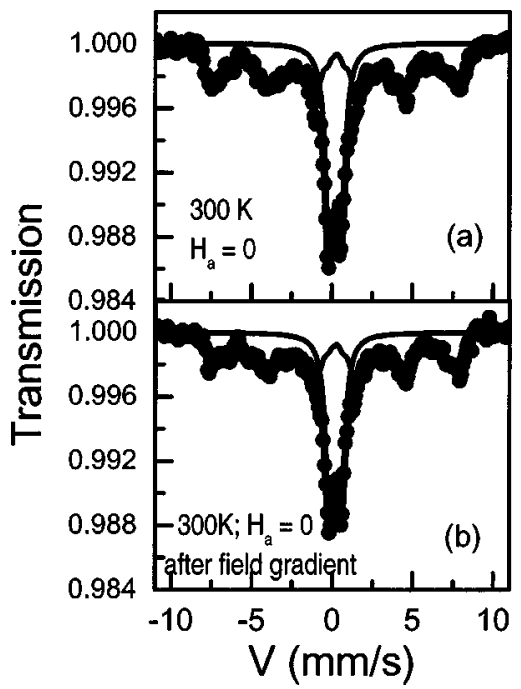

FIG. 7. Mössbauer spectra of sample BR-P at $300 \mathrm{~K}$ in $H_{a}=0$ before (a) and after (b) the magnetic field gradient exposure.

the expected behavior for a soft magnetic material. By comparing spectra of Fig. 6(a) and 6(c), it turns out that after the field treatment, a decrease of $\sim 25 \%$ of the total Mössbauer resonant area occurs (see Table II). Similar results were obtained at $300 \mathrm{~K}$ after carrying out the same procedure (Fig. 7) where a decrease of $10 \%$ of the total resonant area is observed. The area of a Mössbauer spectrum is proportional to the fraction of absorption events taking place without recoil of the nucleus. This fraction $f$ is proportional to the total mean square displacement of the nucleus: $f \alpha \exp \left[-k\left\langle x_{t}^{2}\right\rangle\right]$. As both area and line shape of the Mössbauer spectra can be influenced by dynamical processes, fast motions can in principle remove the resonant area. The lost area could be ascribed to larger mean-square displacements. For example, in ultrafine $\mathrm{Fe}(\mathrm{OH})_{3}$ particles precipitated inside the Dowex 50 $\mathrm{W}$ resin, ${ }^{12}$ a crossover has been observed with Mössbauer spectroscopy from free diffusion in restricted volumes to diffusion of the Brownian oscillator, on increasing the degree of polymer crosslinkage. ${ }^{12}$ In our case, as the degree of crosslinkage is fairly low $(1 \%-2 \%)$, the observed features could be explained in terms of a partial decoupling of particles from their matrix. In other words, as a result of a free diffusion in a restricted volume of a fraction of particles, the system behaves as if there were no barriers preventing their magnetic moments orienting along the external magnetic field. The decrease of the fraction of freely rotating particles with increasing temperature can be explained by two main

TABLE II. Mössbauer resonant areas at $77 \mathrm{~K}$ for sample BR-P according to the following experimental sequence: (i) spectrum recorded in the absence of an applied magnetic field; (iv) zero applied field spectrum recorded after removing the field.

\begin{tabular}{lccc}
\hline \hline & $\begin{array}{c}\text { Total } \\
\text { normalized } \\
\text { resonant area }\end{array}$ & $\begin{array}{c}\text { Superparamagnetic } \\
\text { fraction (\%) }\end{array}$ & $\begin{array}{c}\text { Ordered fraction } \\
(\%)\end{array}$ \\
\hline (i) & 100 & $37 \%$ & $63 \%$ \\
(iv) & 89 & $38 \%$ & $62 \%$ \\
\hline \hline
\end{tabular}

features: (i) the thermal reduction of the Mössbauer recoilless fraction, which is enhanced by the size effects, and (ii) the decreasing number of particles in the blocked magnetic state. Although the thermal energy is expected to weaken the bonds between the particles and the polymeric matrix, it actually counteracts the orienting action of the external magnetic field, leading to the observed decrease of the freely rotating fraction.

To explain all the experimental findings, the structural and morphological characteristics of the nanocomposites have to be considered and the nature of the matrix is also expected to play an important role. In particular, the interactions between the particles and the matrix are probably mediated by water molecules (thermogravimetric analyses for BR-P have shown a weight loss $\sim 40 \%$ below $100{ }^{\circ} \mathrm{C}$ ) forming hydrogen or van der Waals bonds between the particles and the polymeric matrix. ${ }^{13}$ Indeed, after subjecting the particles to the magnetic field treatment, the induced stresses may be sufficient to break some of the weak bonds between the surfactant layer and the matrix wall. In such a case the predominant magnetization process of the decoupled fraction of the particles assembly can be a "nearly free" body rotation as in ferrofluids. ${ }^{14}$ As a consequence, particles can follow small variations of the magnetic fields without time lags and no magnetic relaxation thus occurs.

\section{SUMMARY AND CONCLUSIONS}

Magnetization cycle measurements at $5 \mathrm{~K}$ on iron-oxide nanoparticles in phosphonic-polystyrene type resins, crosslinked with divinylbenzene (BR-P samples), lend support to the existence of a fraction of particles able to rotate freely in response to an applied field, after carrying out a suitable field treatment for decoupling the particles from the matrix. The effect is also observed by means of Mössbauer spectroscopy as indicated by the decrease of the total resonant area. Moreover, it still persists at $77 \mathrm{~K}$ as well as at room temperature, although to a lesser extent.

The free-rotor behavior of a nanomagnet precludes the possibility of magnetic relaxation ${ }^{5}$ and opens up the possibility of almost magnetically lossless materials, ideal for applications in sensors, electrical transformers, motors, and generators.

\section{ACKNOWLEDGMENTS}

This research was supported by the European Community (CRAFT Project, Grant No. NBE-S2-2806) L1.C. wishes to thank the Generalitat de Catalunya for his Ph.D fellowship (No. 1998FI-0005).

${ }^{1}$ G. C. Hadjipanayis, J. Magn. Magn. Mater. 200, 373 (2000); F. Mazaleyrat and L. K. Varga, ibid. 215-216, 235 (2000).

${ }^{2}$ I. Horio, X. Z. Zhou, and A. H. Morrish, J. Magn. Magn. Mater. 118, L279 (1993); J. Phys. Soc. Jpn. 63, 769 (1994).

${ }^{3}$ C. P. Luo and D. Sellmyer, Appl. Phys. Lett. 75, 3162 (1999).

${ }^{4}$ M. Yu, Y. Liu, A. Moser, D. Weller, and D. J. Sellmyer, Appl. Phys. Lett. 75, 3992 (1999).

${ }^{5}$ J. Tejada, X. X. Zhang, E. C. Kroll, X. Bohigas, and R. F. Ziolo, J. Appl. Phys. 87, 8008 (2000).

${ }^{6}$ F. Svec and J. M. J. Frechet, Science 273, 205 (1996). 
${ }^{7}$ L. Suber, S. Foglia, G. M. Ingo, and N. Boukos, Appl. Organomet. Chem. 15, 414 (2001)

${ }^{8}$ P. Ayyub, M. Multani, M. Barma, V. R. Palkar, and R. Vijayaraghavan, J. Phys. C 21, 2229 (1988); N. Randrianantoandro, P. Laffez, C. Sella, and J. M. Greneche, Eur. J. Phys. 9, 125 (2000); N. Randrianantoandro, A. M. Mercier, M. Hervieu, and J. M. Greneche, Mater. Lett. 47, 150 (2001).

${ }^{9}$ J. L. Dormann, D. Fiorani, and E. Tronc, Adv. Chem. Phys. 98, 283 (1997).

${ }^{10}$ G. M. da Costade, E. Grave, P. M. A. de Bakker, and R. E. Vandenberghe, Clays Clay Miner. 43, 656 (1995); S. Mørup and H. Topsøe, J. Magn.
Magn. Mater. 31-34, 953 (1983).

${ }^{11}$ E. Murad, Phys. Chem. Miner. 23, 248 (1996).

${ }^{12}$ A. S. Plachinda, V. E. Sedov, V. I. Khromov, L. V. Bashkeev, and I. P. Suzdalev, Hyperfine Interact. 56, 1483 (1990); A. S. Plachinda, V. E. Sedov, V. I. Khromov, L. V. Bashkeev, I. P. Suzdalev, V. I. Goldanskii, G. U. Nienhaus, and F. Parak, Phys. Rev. B 45, 7716 (1992).

${ }^{13}$ P. V. Hendriksen, S. Morup, and S. Linderoth, J. Phys. C 3, 3109 (1991).

${ }^{14} \mathrm{P}$. Scholten, in Magnetic Properties of Fine Particles, edited by J. L. Dormann and D. Fiorani (North-Holland, Amsterdam, 1992), pp. $277-286$. 